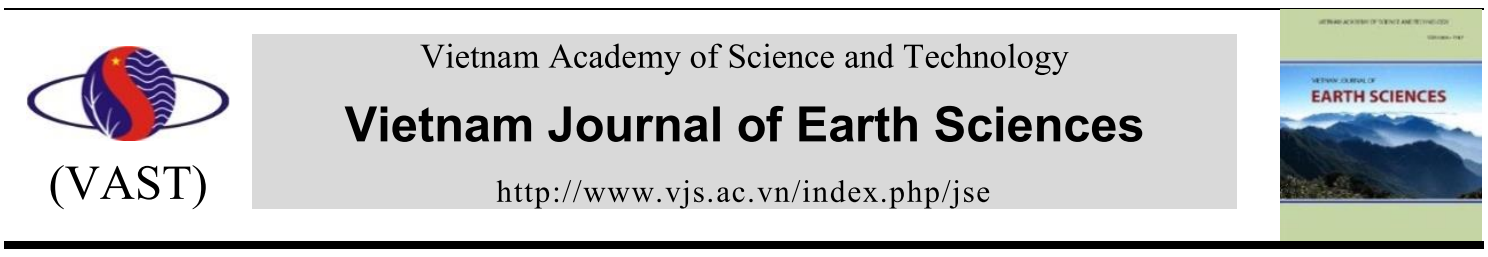

\title{
Variation of the foF2 parameter during fluctuating activity: Prediction with IRI-2012 compared to measured data from Ouagadougou inosonde station during solar cycles 21 and 22
}

\author{
Abidina Diabaté ${ }^{1}$, Jean Louis Zerbo ${ }^{1,2^{*}}$, Frédéric Ouattara ${ }^{1}$ \\ ${ }^{1}$ Laboratoire de Recherche en Energétique et Météorologie de l'Espace (LAREME) Université Norbert \\ Zongo, BP 376, Koudougou, Burkina Faso \\ ${ }^{2}$ UFR/ST-Université Nazi Boni, Bobo Dioulasso, 01 BP 1091 Bobo, Burkina Faso
}

Received 30 November 2018; Received in revised form 29 December 2018; Accepted 9 January 2019

\begin{abstract}
In this paper, we review on diurnal variations of the foF2 ionospheric parameter predicted by the IRI-2012 model, and data from Ouagadougou ionosonde station located in the crest of the Equatorial Anomaly (Lat: $12.5^{\circ} \mathrm{N}$; Long: $358.5^{\circ} \mathrm{E}$, dip: $1.43^{\circ}$ ) during fluctuating geomagnetic activity conditions for the solar cycles 21 and 22 . Our investigations are focused on the electrodynamic aspects, the influence of the ionospheric electric currents as well as the variations of the hourly values given by model and experimental measurements. A comparative study pointed out that the IRI-2012 model, through its URSI and CCIR subroutines, gives a good prediction of the critical frequency of the F2 layer between $0700 \mathrm{TL}$ and $0000 \mathrm{TL}$. In addition, IRI -2012 tries to reproduce, as best as possible, the vertical drift $\mathrm{E} \times \mathrm{B}$ during minimum, decreasing phase, winter, and autumn. However, there is no effect of drift during the other seasons and solar cycle phases. A last, the model does not take into account the PRE phenomenon observed in autumn and the influence of the equatorial electrojet in this ionospheric zone.
\end{abstract}

Keywords: Fluctuating activity; solar cycle phase; seasons; F2 layer critical frequency; deviation percentage; electrojet; Reversal.

C2019 Vietnam Academy of Science and Technology

\section{Introduction}

The F2 layer is the most important layer of the ionosphere for the propagation of radio waves in the high-frequency band due to its height and electron density. One of the appropriated parameters used to get a global overview of this ionospheric layer is its critical frequency foF2 very important for telecommunication. To achieve this goal,

"Corresponding author, Email: jeanlouis.zerbo@gmail.com empirical models of prediction have been developed. One of them is International Reference Ionosphere (IRI) developed in the 1960s by the Committee on Space Research (COSPAR) and International Union of Radio Science (URSI). Its goal is to establish an international standard for fundamental ionospheric parameters (Bilitza and Reinisch, 2007). Over time, the model has been progressively improved with more recent data and better mathematical descriptions. Today, 
IRI is internationally accepted as a standard for ionospheric parameters and is used by many authors (Bilitza and Reinisch, 2007; Oyekola and Fagundes, 2012; Bilitza et al., 2014; Ouattra and Nanéma 2014). Its 2012 version allows the determination of seven important parameters including the critical frequency foF2. Many comparative studies between the ionospheric parameters from the model and those measured in stations have helped to appreciate IRI-model different versions and to improve them. Investigation at Ouagadougou ionosphere station in Burkina Faso (Lat: $12.5^{\circ} \mathrm{N}$, Long: $358.5^{\circ} \mathrm{E}$, dip: $1.43^{\circ}$ ) may contribute to the improvement of ionospheric parameters prevision in the equatorial zone. Certain authors (Adeniyi1 et al., 2005; Oyekola and Fagundes, 2012; Ouattra and Nanéma 2014; have reviewed on the similar subject. Our present work investigates on foF2 parameter variations obtained from the 2012 IRI model and in situ measurements carried out at the Ouagadougou station for fluctuating solar wind conditions during the solar cycles 21 and 22.

The present study goal is to identify points of agreement and possible disparities between the values observed and predicted values of foF 2 in order to contribute to an improvement of the model in the equatorial region. The document is structured in 6 sections: In section 2 we present the data as well as the analysis methods used. In section 3 we expose the results obtained. These results are discussed in Section 5 followed the conclusion.

\section{Data and methods}

\subsection{Data}

The ionospheric parameter studied is the critical frequency of the F2 layer (foF2).

The hourly values of foF 2 are taken from Ouagadougou inosonde station (Lat: $12.5^{\circ} \mathrm{N}$, Long: $358.5^{\circ} \mathrm{E}$, dip: $1.43^{\circ}$ ) in Burkina Faso. These values come from the database of
Télécom Bretagne (ENST Bretagne). Period cover by our investigation is 1976 to 1996 , corresponding to solar cycles 21 and 22 .

The foF 2 hourly predicted values are carried out by using the IRI-2012 model (ttp://omniweb.gsfc.nasa.gov/vitmo/iri2012)

through its two subroutines URSI (International Union of Radio Science) and CCIR (International Consultative Committee of Radio Communications).

The values of sunspots $\mathrm{Rz}$ are obtained from the SPIDR database (URL http://spidr.ngdc.noaa.gov/spidr/).

The geomagnetic index aa are from http://isgi.unistra.fr/data download.php and summarized in pixel diagram (Figure 1, an example for the year 1976) and fully described by (Legrand and Simon, 1989; Ouattara and Amory-Mazaudier , 2009; Zerbo et al., 2012; Zerbo et al., 2013).

\subsection{Methods}

Solar cycle phases are determined by using sunspot number $\mathrm{Rz}$ and criteria (Zerbo et al. 2011; Ouattra, 2013): (1) the minimum phase: $\mathrm{Rz}<20$; (2) the ascending phase: $20 \leq \mathrm{Rz} \leq$ 100 and $\mathrm{Rz}$ greater than the previous year's value; (3) the maximum phase: $\mathrm{Rz}>100$; (4) the decreasing phase: $100 \geq R z \geq 20$ and $\mathrm{Rz}$ less than the previous year values.

Seasons are identified as followed: winter (December, January, and February); spring (March, April, May); summer (June, July, August) and autumn (September, October and November).

Days under fluctuating geomagnetic activity are selected through the geomagnetic activity classification (Legrand and Simon, 1989). According to this classification, (1) the quiet activity corresponds to the days with index $\mathrm{Aa}<20 \mathrm{nT}$, (2) the recurrent activity groups the days with index $\mathrm{Aa} \geq 40 \mathrm{nT}$ on at least one rotation, (3) the shocks activity is characterized by dates of SSC with the index $\mathrm{Aa} \geq 40 \mathrm{nT}$ for a duration not more than three 
days. The fluctuating activity includes all the

class of activity. Figure 1 is an illustration of other days not identified in the three previous the different classes of geomagnetic activity.

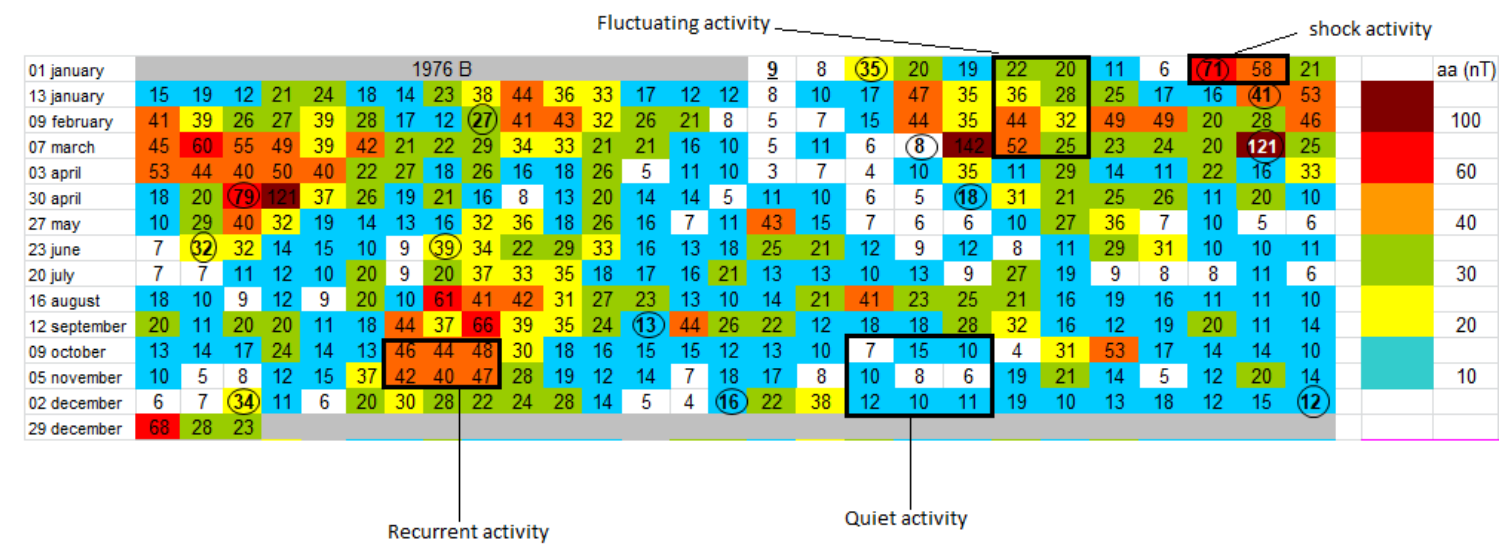

Figure 1. Pixel diagram of the year 1976 illustrating geomagnetic activity classes. Each line shows solar rotation, successive lines of solar rotations, and each number the daily average of solar wind speed. The circle indicates the date of storm/coronal mass ejection (CME)

The deviation percentage (DP) help to quantify the difference between the predicted values and those observed. It is obtained by:

$$
D P=\frac{x_{i}^{m}-x_{i}^{o}}{x_{i}^{o}} \times 100
$$

Where $x_{i}^{0}$ et $x_{i}^{m}$ respectively represent the observed and modeled foF 2 values.

$D P>0$ : The model overestimates the value of foF2;

$D P<0$ : The model underestimates the value of foF 2 .

To appreciate the morphological or qualitative behavior of the predicted foF2 values, we use the error bars carried on the observed foF2 profile through $\sigma=\sqrt{\mathrm{V}}$; where $\mathrm{V}$ is the variance defined by $\frac{1}{\mathrm{~N}} \sum_{\mathrm{i}=1}^{\mathrm{N}}\left(\mathrm{x}_{\mathrm{i}}-\overline{\mathrm{x}}\right)^{2}$ with $\overline{\mathrm{x}}$ mean value and $\mathrm{N}$ the total number of available data).

\section{Results and Analysis}

\subsection{Comparison per solar phase}

Figure 2 shows the diurnal variations of foF2 (left panels) and deviation percentages (right panels) for the minimum (panels a), the ascending phase (panels $b$ ), the maximum (panels c) and the decreasing phase (panels d) of soalr cycle. Measured data from Ouagadougou inosonde station data are represented by solid line while the IRI predicted values are in dash and dotted lines for the URSI and CCIR subroutines respectively.

At solar minimum (Fig. 2A, left panel) predicted and measured values of foF 2 show an increasing trend: from $0500 \mathrm{LT}$ to $1800 \mathrm{LT}$ for inosonde measurement and CCIR prediction; and between $0400 \mathrm{LT}$ and $16 \mathrm{~h} 00$ LT for URSI. The profile of the data shows an afternoon peak at $1800 \mathrm{LT}$ and a slight dip around 1200 LT. Afternoon peaks are also observed with IRI predictions at 1600 and 1800 for URSI and CCIR respectively. However, the midday trough is not taken into account by IRI forcastings. The deviation percentage (Fig. 2A; right panel) shows values within $\pm 10 \%$ for the URSI version between 08:00 LT and 0100 LT and values in the range of $\pm 05 \%$ between 0600 and 00:00 LT for CCIR version. From 0000 LT to 06:00 LT, DP profiles deviate significantly from the zero value. 
Abidina Diabaté et al./Vietnam Journal of Earth Sciences 41(2019)

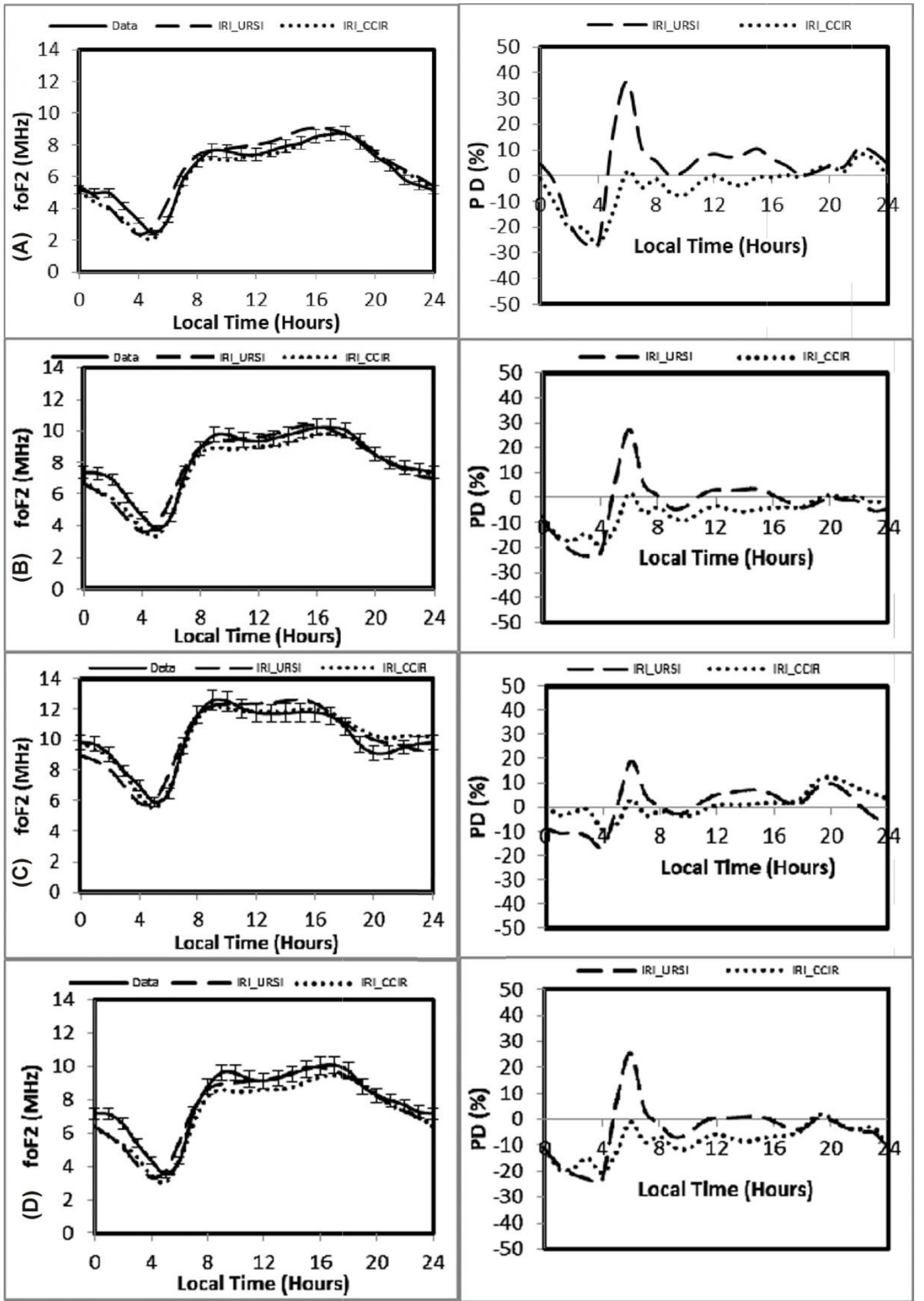

Figure 2. Observed and modeled diurnal variations of foF2 (left) and deviation percentage (right) per solar phases during fluctuating activity period 1976 to 1996 
Vietnam Journal of Earth Sciences, 41(1), 59-68

With URSI one can see a negative peak $(-26.05 \%)$ at $04: 00$ and a positive peak one $(36.56 \%)$ at 00600 and with CCIR on can remark only a negative peak $(-26.84 \%)$ at 0400 LT. The percentages of deviation are negative with CCIR most of the time except between 1900 and 23:00. The reverse is observed with the URSI version where we observe positive values from 05:00 to 00:00 LT.

During the increasing phase of the solar cycle, the profile of the data (Figure 2B; left panel) does not show any particular trend; however, both versions of IRI show slight increasing trends between 10:00 LT and 17:00 LT. We observe a "noon bit out" profile with the recorded data; the first peak at 09:00 LT and the second at 17:00 LT. The IRI models indicate afternoon peak profiles, the peak occurring respectively at 15:00 LT and 16:00 LT for URSI and CCIR.

The right panel of Fig. 2B shows that deviation percentages are most of the time in the order of $\pm 10 \%$ from 07:00 LT up to 00:00 LT with the URSI version and from 06:00 LT to 00:00 LT with the CCIR version. URSI version presents a negative peak at 03:00 LT with a DP $=-23.41 \%$ and a positive peak at 06:00 with a $\mathrm{DP}=27.27 \%$. CCIR records a negative peak at 04:00 LT with a DP= $-19.15 \%$. From $17: 00$ to $04: 00$ LT, the deviation percentages obtained with URSI are negative. The percentages of deviations are also negative with CCIR, except at 06:00 LT; and between 20:00 and 23:00 LT.

At solar maximum, the IRI prediction (Fig. 2C; left panel) shows a "plateau" profile when in situ data present a "morning peak" observed at 09:00 LT and a trough around 20:00 LT. The CCIR version tries to reproduce the trough at the same time, however, the trough is much less important. The URSI deviation percentage (Figure 2C; right panel) shows values in the range of \pm 10\%, except between 0100 LT and 04:00 LT where they vary between 10 and $16 \%$. For CCIR, DP's values are in the order of $\pm 05 \%$.
With the URSI version, the negative percentages are recorded between 22:00 LT and $0400 \mathrm{LT}$ (where DP $=16.22 \%$.) and with CCIR they are between 01:00 LT and 11:00 LT.

During the decreasing phase (Figure 2D; left panel) an "afternoon peak" is observed with IRI versions predictions and a double peak in the inosonde data. The peaks in measured data occur respectively at 09:00 LT and 17:00 LT; and a trough is observed around 12:00 LT. Here again CCIR version tries to fairly reproduce a trough around 13:00 LT.

Figure 2D (right panel) shows that the deviation percentage values are between \pm 10\% from 07:00 LT to 23:00 LT. Especially, between 11:00 LT and 22:00 LT, the URSI percentages are confined at $\pm 05 \%$. The percentages of deviation are negative with URSI from 1600 LT 0400 LT. The deviation percentages of CCIR are negative most of the time except between 19:00 LT and 20:00 LT. The URSI version, deviation percentages profile shows a negative peak at 04:00 LT with DP $=-23.64 \%$ and a positive peak at 06:00 LT with DP $=+25.38 \%$. The highest DP with CCIR is $+20.71 \%$, observed at 04:00 LT.

\subsection{Comparison per season}

In this section, the seasonal diurnal variations of foF 2 values predicted with URSI and CCIR are compared with those recorded at the Ouagadougou inosonde station. Figure 3 shows the results obtained for different seasons. The left panels give the diurnal profiles of foF 2 and those on the right show the corresponding deviation percentages when CCIR or URSI are used. From top to bottom, we have winter (panels a), spring (panels b), summer (panels c) and autumn (panels d). Data from Ouagadougou inosonde station are represented by solid lines while the IRI predicted values are in the dash and dotted lines for the URSI and CCIR sub-routines respectively. 
During winter (Fig. 3A, left panel), the observed foF2 has a "noon bite out"profile. The first peak occurs at 10:00 TL while the second peak, much less important, occurs at 16:00 LT. IRI predictions give a "plateau" profile for both subroutines (CCIR and URSI), with a slight trough observed around 14:00 TL. There is no trend at the data level while the model indicates an increasing trend in foF2 between 09:00 TL and 17:00 TL.

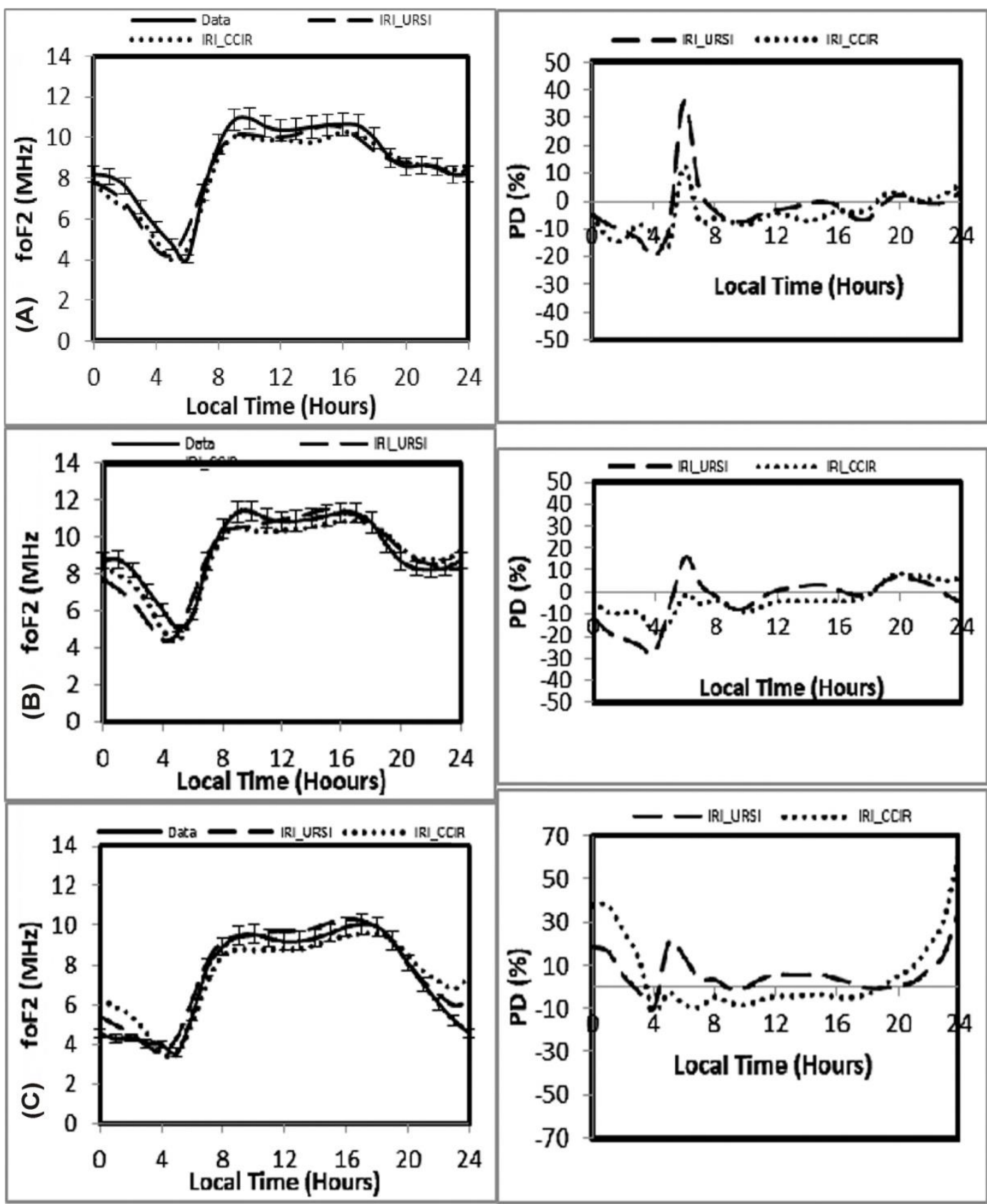

Figure 3. Observed and modeled seasonal diurnal variations of foF2 (left) and deviation percent (right) under fluctuating activity conditions during solar cycles $21-22$ 
From 07:00 to 00:00 TL, the deviation percentage (Fig. 3A, right panel) fluctuates between $\pm 05 \%$, with some percentages reaching $\pm 08 \%$. Between 02:00 TL and 06:00 TL we observe important two deviation percentages; a positive peak $(+35.82 \%)$ observed with URSI at 06:00 TL and a negative peak $(-16.37 \%)$ with CCIR at 05:00 TL. Both curves are under zero except between 05:00 TL and 06:00 TL and after 18:00 TL up to 24:00 TL.

In spring (Fig. 3b, left panel), we observe a double peak in the data while the model shows an "afternoon" peak profile with URSI and a "plateau" profile with CCIR. Between 09:00 TL and 17:00 TL we note an increasing trend in IRI values.

For the PD variations (Fig. 3B, right panel), the values are in the range of $\pm 10 \%$ from 07:00 TL to 24:00 TL for URSI and from 0600 to 03:00 TL for CCIR. The URSIversion presents a peak $(-21.20 \%)$ at $04: 00$ and another at 06:00 TL (15.99\%). The DP of CCIR is negative from 00:00 LT to 18:00 TL and reverses after 18:00 while DP with URSI is always fluctuating between positive and negative values.

During summer (Fig. 3C, left panel), an afternoon peak is observed with predictions when in situ measurements are characterized by a double peak. In addition, a slight dip is observed during the night (around 23:00 TL) on IRI-model predictions.

The deviation percentages in summer (Fig. $3 \mathrm{C}$, right panel) are between $\pm 10 \%$ from 07:00 TL to 21:00 TL for URSI and from 05:00 TL to 20:00 TL for CCIR. One can see that DP profile deviates significantly from zero from 22:00 to 01:00 TL for URSI and 03:00 TL for CCIR. Except for the midnight peak, $34.94 \%$ and $61.70 \%$ are recorded respectively with URSI and CCIR, the two curves are mostly above zero.

Autumn (Fig. 3D, left panel) is characterized by a double peak in data and a trough around 12:00 TL. The modeled profiles show a "plateau" profile. In addition, the data profile shows another trough at 20:00 TL followed by a peak of around 21:00 LT.

Between 07:00 TL and 00:00 TL, the percentages of deviations (Fig. 3D, right panel) are between $\pm 06 \%$. High values of DP are observed between 01:00 TL and 06:00 TL. The URSI version shows a negative peak $(-21.06 \%)$ at $04: 00$ TL and another peak $(+21.47 \%)$ at $06: 00 \mathrm{TL}$. The CCIR gives negative values from 00:00 TL to 20:00 TL and positive values starting at 22:00 TL.

A simple comparison between foF 2 profiles during the different seasons shows that profiles during the equinoxes are always above those of the solstices for in situ data and IRI predictions. However, we find that at equinoxes, from 09:00 TL to 19:00 TL, in situ foF 2 profiles during spring are greater than those of autumn; the opposite is observed with the model.

\section{Discussions}

In this section, we review on the following comparison criteria: (a) Quantitative analysis of the difference between observed and predicted foF 2 values through the deviation percentages (DP). (b) The influence of the electric field on the foF2 through the equatorial electrojet. Many works (Faynot and Villa, 1979; Vassal, 1982; Acharya et al. 2010; Acharya et al. 2011) teach about the possible link between the different foF 2 profiles and the presence or absence of the electrojet. Thus a "noon bite-out" profile is characteristic of the existence of an electrojet and a counter-electrojet, respectively in the morning and in the afternoon; a "morning peak" profile indicates the existence of a moderate electrojet during the day, a "Dome" profile corresponds to the absence of electrojet. An "afternoon peak" profile induces an intense counter-electrojet during the afternoon and a "Plateau" profile shows 
the presence of a weak electrojet during the day. (c) the vertical drift of the layer F2. According to Farley et al. (1986), Fejer (1981), Fejer et al. (1979), and Rishbeth(1971), the signature of the drift is materialized by the existence of the midday trough in the foF 2 profile. (d) The representation of the post-sunset peak that is characteristic of the inversion of the magnetic field in the equatorial ionosphere identified by "pre-reversal enhancement" (PRE) n many investigations. (e) The effect of the season on the variation of foF2. Indeed, the position of the Ouagadougou station regardless with the Equatorial Ionospheric Anomaly argues the fact that seasons have an impact on foF2 behavior (Obrou, 2008).

Through the deviation percentage one can note that for all the solar phases and for all the seasons, the percentages are confined between $\pm 10 \%$ from $07: 00$ to $00: 00 \mathrm{TL}$. This result shows that more than $70 \%$ of the hourly values of foF 2 predicted by IRI (URSI and CCIR) are in good correlation with the values observed. Using the relative standard deviation, a similar result was obtained for the IRI-2005 previsions (Adeniyi et al. 2005). But, the largest differences are observed between 01:00 and 040:0 TL where the URSI version has the highest percentages with peaks of up to $\pm 30-40 \%$. We can argue that both subroutines (CCIR and URSI) give acceptable foF2 values during the post-sunrise period up to 00:00 TL. However, during the pre-sun period, the foF 2 values predicted by the model do not agree with in situ data taken from Ouagadougou inosonde station.

Investigations on the different profiles show that the electric currents signature, characteristic of the equatorial ionosphere, is not sufficiently taken into account the present IRI version. In fact, the in situ data show a double peak during the ascending and decreasing solar cycles phases, an afternoon peak and a morning peak respectively during the minimum and maximum solar cycle phases. The model predicts a plateau profile during the solar maximum phase and "afternoon peak" profiles during other phases. The same observation is given with the seasonal profiles: the experimental values show a double peak during all the seasons while the model presents an afternoon profile in summer and a "plateau" profile during the other seasons.

From the electrodynamic point of view, we observe an absence of midday trough in the model predictions. Notwithstanding, during certain periods (minimum and descending solar phases, winter and autumn) the model tries to reproduce the trough. Thereby, these results show that the IRI model does not produce very well the signature of the vertical drift.

The night peak resulting from the effects of PRE of the electric field is one of the characteristics of the impact of fluctuating activity in autumn (Ouattara, 2009). In our study, this phenomenon is actually observed in autumn with recorded data only. That means that IRI model does not reproduce the PRE.

One can argue that data and model take into account some seasonal properties of the variations of the critical frequency: the values of foF 2 during the equinoxes are higher than those of the solstices. However, the equinoctial properties are not reproduced by IRI-2012 version for equatorial region.

\section{Conclusions}

We have studied the diurnal variations of the critical frequency of ionosphere F2 layer (foF2) predicted by the IRI-2012 model, compared to the experimental data recorded at the Ouagadougou inosonde station under the fluctuating geomagnetic activity conditions over solar cycles 21 and 22. Our investigations show that the IRI-2012 model provides foF2 values very close to the 
observed values, especially between sunrise and midnight. In addition, the model attempts to reproduce the vertical drift during certain periods of solar activity (minimum and decreasing phase) and during certain seasons (winter and autumn). It has been also noted that the model takes into account some seasonal properties of the foF 2 parameter. However, there are some shortcomings in the model predictions: (1) During the pré-sunrise period, the predicted foF 2 values deviate considerably from the observed values, (2) the vertical drift and the electrojet equatorial are not taken into account by the model, (3) the model does not predict the pre-inversion phenomenon which is one of the characteristics of fluctuating activity during autumn.

These results show the necessity to improve this IRI version (URSI and CCIR) by integrating parameters that take into account the effects of physical processes such as $\mathrm{E} \times \mathrm{B}$ drift, ionospheric electric currents (the electrojet), the pre-inversion phenomenon.

The largest deviations between the predicted values and those observed are seen before sunrise. Since the sun is the main source of ionization of the ionosphere, we think that efforts must be done to take into account solar radiation during this period to reduce the differences between the measurements and the model predictions.

\section{Acknowledgments}

The authors thank Brest Telecom for providing Ouagadougou inosonde data and ISGI data centre for providing the aa indices.

\section{References}

Acharya R., Roy B., Sivaraman M.R., 2010. Dasgupta A. An empirical relation of daytime equatorial total electron content with equatorial electrojet in the Indian zone. J Atmos Terr Phys, 72(10), 774-780.

Acharya R., Roy B., Sivaraman M.R., Dasgupta A., 2011. On conformity of the EEJ based Ionospheric model to the Fountain effect and resulting improvements. J Atmos Terr Phys, 73, 779-784.

Adeniyi J.O., Oladipo O.A., Radicella S.M., 2005. Variability of fof 2 and comparison with iri model for an equatorial station. The Abdus Salam International Centre for Theoretical Physics, IC/2005/085, http://www.ictp.it/ pub off.

Adeniyi1 J.O., Oladjipo O.A., Radicella S.M., 2005. Variability of foF2 and comparison with IRI model for an equatorial station. The Abdus Salam International Centre for Theoretical Physics, IC/2005/085.

Bilitza D., et al., 2014. The International Reference Ionosphere 2012-a model of international collaborationI. J. Space Weather Space Clim, 4, A07.

Bilitza D., Reinisch B.W., 2008. International Reference Ionosphere 2007: Improvements and new parameters. Adv. Space Res, 42, 599-609.

Farley D.T., Bonell E., Fejer B.G., Larsen M.F., 1986. The Prereversal Enhancement of the Zonal Electric Field in the Equatorial Ionosphere. J Geophys Res, 91(A12), 13,723-13,728.

Faynot J.M., Villa P., 1979. F region at the magnetic equator. Ann Geophys, 35, 1-9.

Fejer B.G., 1981. The equatorial ionospheric electric fields: A review. J Atmos Terr Phys, 43, 377.

Fejer B.G., Farley D.T., Woodman R.F., Calderon C., 1979. Dependence of equatorial $F$ region vertical drifts on season and solar cycle. J Geophys Res, 84, 5792.

Legrand J.P., Simon P.A., 1989. Solar cycle and geomagnetic activity: A review for geophysicists. Part I. The contributions to geomagnetic activity of shock waves and of the solar wind. Ann. Geophys, 7, 565-578.

Obrou K.O., 2008. Contribution à l'amélioration du modèle "International Reference Ionosphere" (IRI) pour l'ionosphère équatoriale. Thèse de doctorat Université de Cocody, Abidjan, Côte d'Ivoire.

Ouattara F., 2009. Contribution à l'étude des relations entre les deux composantes du champ magnétique solaire et l'Ionosphère Equatoriale. Thèse de Doctorat d'Etat ès Sciences, Université Cheikh Anta Diop, Dakar, Sénégal. 
Abidina Diabaté et al./Vietnam Journal of Earth Sciences 41(2019)

Ouattara F., 2013. IRI-2007 foF2 Predictions at Ouagadougou Station during Quiet Time Periods from 1985 to 1995. Archives of Physics Research, 4, $12-18$.

Ouattara F., Amory-Mazaudier C., 2009. Solargeomagnetic activity and $\mathrm{Aa}$ indices toward a Standard. J. Atmos. Terr. Phys, 71, 1736-1748.

Ouattra F., Nanéma, 2014. Quiet Time foF2 Variation at Ouagadougou Station and Comparison with TIEGCM and IRI-2012 Predictions for 1985 and 1990. Physical Science International Journal, 4(6), 892-902.

Oyekola O.S., Fagundes P.R., 2012. Equatorial F2-layer variations: Comparison between F2 peak parameters at Ouagadougou with the IRI-2007 model. Earth, Planets Space, 64, 553-566.

Rishbeth H., 1971. The F-layer dynamo. Planet, Space Sci, 19, 263.
Vassal J.A., 1982. The variation of the magnetic field and its relationship with the equatorial electrojet in Senegal Oriental. Annals of Geophysics, Tome French, 38.

Zerbo J.L., Amory-Mazaudier C. Ouattara F., Richardson J., 2012. Solar Wind and Geomagnetism, toward a Standard Classification 1868-2009. Ann Geophys, 30, 421-426. http://dx.doi.org/10.5194/angeo-30-421-2012.

Zerbo J.L., Amory-Mazaudier C., Ouattara F., 2013. Geomagnetism during solar cycle 23: Characteristics. J. Adv. Res, 4(3), 265-274. Doi:10.1016/j.jare.2013.08.010.

Zerbo J.L., Ouattara F., Zoundi C., Gyébré A., 2011. Solar cycle 23 and geomagnetic activity since 1868 . Revue CAMES serie A, 12(2), 255-262. 